\title{
EFFECT OF INTRAVESICAL PROSTATIC PROTRUSION (IVPP) ON LOWER URINARY TRACT FUNCTION AND MANAGEMENT
}

\author{
1 Professor \& HOD, Department of Urology, Government Stanley Hospital, Chennai. \\ ${ }^{2}$ Associate Professor, Department of Urology, Government Stanley Hospital, Chennai. \\ ${ }^{3}$ Assistant Professor, Department of Urology, Government Stanley Hospital, Chennai. \\ ${ }^{4}$ Assistant Professor, Department of Urology, Government Stanley Hospital, Chennai. \\ 5Junior Resident, Department of Urology, Government Stanley Hospital, Chennai. \\ 6 Junior Resident, Department of Urology, Government Stanley Hospital, Chennai. \\ 7 Junior Resident, Department of Urology, Government Stanley Hospital, Chennai. \\ 8Junior Resident, Department of Urology, Government Stanley Hospital, Chennai.
}

Rajaraman T1, Balaji A. R2, Vetrichandar $S^{3}$, Shekar M. G4, Bhat K. R. $S^{5}$, Muthurathinam $R^{6}$, Ashokkumar $R^{7}$, Lakshminarayan K. $R^{8}$

\section{ABSTRACT}

\section{AIMS AND OBJECTIVES}

To correlate Intravesical Prostatic Protrusion (IVPP) with International Prostate Symptoms Score (IPSS), Quality of Life Index (QOL), Uroflowmetry, Post Void Urine (PVR) and Pressure Flow Study (PFS) and to assess whether the presence and increasing grades of Intravesical prostatic protrusion are directly correlated with the severity of Bladder outlet obstruction due to Benign prostatic hypertrophy.

\section{MATERIALS AND METHODS}

Non-randomized prospective cohort study, conducted in Department of Urology, Govt. Stanley Hospital, from June 2012 to June 2015, on a sample of 100 patients with IVPP. IVPP was correlated with IPSS, Quality of life index, Uroflowmetry, Effects of drug therapy, Response to surgical therapy. Response of patients with Intravesical prostatic protrusion of same grade to surgical therapy and drug therapy are compared to decide which modality of management is best.

\section{RESULTS}

The incidence of IVPP in patients presenting with LUTS due to BPH is 1 in 5. Majority present with grade 2 IVPP. The increasing grades of IVPP are significantly directly correlated with IPSS score, Q-max and Post void residual. Statistical analysis showed IVPP to correlate significantly with BOO. TURP showed a very good and significant mean decrease of IPSS and Q-max, whereas those who had medical treatment showed only a mean decrease of 2 in IPSS score and 1 in Q-max.

\section{CONCLUSION}

The increasing grades of IVPP are significantly directly correlated with IPSS score max and Post void residual. IVPP have a very good positive and negative predictive value and also a good accuracy rate in comparison with all other parameters in predicting the B00. The surgical intervention statistically proved to be superior to medical treatment in IVPP patients.

\section{KEYWORDS}

IVPP, Prostate, Urology.

HOW TO CITE THIS ARTICLE: Rajaraman T, Balaji AR, Vetrichandar S, et al. Effect of intravesical prostatic protrusion (IVPP) on lower urinary tract function and management. J. Evolution Med. Dent. Sci. 2016;5(55):3796-3800, DOI: 10.14260/jemds/2016/869

\section{INTRODUCTION}

Intravesical Prostatic Protrusion (IVPP) is represented as a severe parameter of the Bladder Outlet Obstruction (BOO) due to Benign Prostatic Hypertrophy (BPH) traditionally and it is considered based on the experience that it can be cured only by surgical management. But till date it is not made standard in various association guidelines and in standard textbooks except for a few publications in literature. In symptomatic benign prostatic hypertrophy patients, there is no consensus or clear practical guidelines to define the presence and severity of obstruction, other than the pressure-flow study. The latter has been traditionally regarded as the 'reference'

Financial or Other, Competing Interest: None.

Submission 31-03-2016, Peer Review 27-04-2016,

Acceptance 03-05-2016, Published 11-07-2016.

Corresponding Author:

Dr. Balaji A. R,

Department of Urology,

NSB $6^{\text {th }}$ Floor, Government Stanley Hospital,

Chennai-1.

E-mail: balaji_ur@yahoo.co.in

DOI: $10.14260 /$ jemds $/ 2016 / 869$ gold standard, but the technique is invasive, uncomfortable for the patient, time-consuming and expensive and not available, especially in most developing countries.

The intravesical prostatic protrusion may not be present in all cases of benign prostatic hypertrophy and hence cannot be accomplished as a single non-invasive measure for assessing the severity of Bladder outlet obstruction due to Benign prostatic hypertrophy. But whomsoever is having Intravesical prostatic protrusion may be considered to be suffering from severe Bladder outlet obstruction and may be taken up for surgical intervention rather than observing with medical management. ${ }^{1}$

\section{AIM OF THE STUDY}

To correlate Intravesical Prostatic Protrusion (IVPP) with International Prostate Symptoms Score (IPSS), Quality of Life Index (QOL), Uroflowmetry.2, Post Void Urine (PVR) and Pressure Flow Study (PFS) and to assess whether the presence and increasing grades of Intravesical prostatic protrusion are directly correlated with the severity of Bladder outlet obstruction due to Benign prostatic hypertrophy. 
Response of patients with Intravesical prostatic protrusion of same grade to surgical therapy and drug therapy are compared to decide which modality of management is best.

\section{MATERIALS AND METHODS}

1. Prospective study.

2. Period of study is from June 2012 to June 2015.

3. Age range 50 to 80 years.

4. The sample size is 100 .

5. The patients with comorbid illness such as Diabetes Mellitus (DM), Hypertension (HTN), Tuberculosis (TB), etc. and associated neurological conditions are excluded from the study. Patients with a known history of previous lower urinary tract surgery, prostate or bladder carcinoma, bladder calculi or neurological deficit are also excluded from the study.

\section{Intravesical Prostatic Protrusion (IVPP) is Correlated with:}

1. International Prostate Symptoms Score (IPSS).

2. Quality of Life Index (QOL).

3. Uroflowmetry.

4. Pressure Flow Study (PFS).

5. Effects of surgical therapy.

6. Response to drug therapy.

The initial evaluation consisted of the International Prostate Symptoms Score (IPSS) and Quality-Of-Life (QUOL) score, a physical examination including a DRE to exclude tumour and a neurological examination to exclude any neurological deficit and neurologically related bladder dysfunction.

The bladder is next assessed by Transabdominal Ultrasonography (TAUS). A grading system is used with three grades depending on the degree of IPP by measuring the vertical distance from the tip of the protrusion to the circumference of the bladder at the base of the prostate gland. ${ }^{3}$

Intravesical prostatic protrusion (IVPP) is graded into three with Transabdominal Ultrasound (TAUS). ${ }^{4}$ with the bladder volume of 150-200 mL.

Grade $\mathrm{I}<5 \mathrm{~mm}$.

Grade II - 5 to $10 \mathrm{~mm}$.

Grade III $>10 \mathrm{~mm}$.

After the Transabdominal Ultrasound (TAUS).5,6 assessment, the peak urinary flow rate ( $Q$ max) and voided volume are measured using Uroflowmetry and the Post Void Residual urine volume (PVR) is measured by diagnostic ultrasonography of the bladder either using the double mode in ultrasound or using the formula $\mathrm{L}^{*} \mathrm{~B}^{*} \mathrm{H}^{*} 0.0523$ considering the spherical shape. Prostate volume is measured by diagnostic ultrasonography of the bladder either using the double mode in ultrasound or using the formula $\mathrm{L}^{*} \mathrm{~B}^{*} \mathrm{H}^{*} 0.0523$ considering the spherical shape.

Urine analysis and culture are done. Patients with positive cultures are treated with appropriate antibiotics and rendered culture free before proceeding with the protocol.

Renal Function test is done and patients with renal insufficiency are excluded.

PSA was also measured and those who had higher values are excluded from the study.
Cystoscopy is done routinely in all patients even though not indicated in all patients according to the standard guidelines after getting the consent.

The cases enrolled in the study after this period underwent routinely the Pressure Flow study using the Delphi's TM Laborie urodynamic machine. BOO was defined by the BOO index (detrusor pressure at Qmax- (2* Qmax)) and then correlated with the clinical variables including age, IPSS7,8, QoL, Qmax, PVR, prostatic volume and IPP grade; the sensitivities and specificities of these variables were then calculated. Logistic regression and Pearson correlation were used for the statistical analysis and correlation coefficient was calculated for the significance.

The Intravesical prostatic protrusion is correlated with the severity of Bladder outlet obstruction due to BPH as assessed by symptoms score, QOL index, Uroflowmetry, cystoscopy and confirmed with Pressure Flow study.

Those patients who are fit and willing for surgery and those patients indicated for surgery are proceeded with conventional TURP.

Other patients are put under drug treatment - a combination of Tamsulosin and Dutasteride for a period of 3 months. The patients who failed medical treatment are planned to undergo surgery after a period of 2 weeks and are observed whether they succeed in voiding or not.

This is a non-randomized prospective cohort study compared on the respective grades and the treatment is said to be effective and complete when Uroflowmetry Peak flow $>15 \mathrm{~mL} / \mathrm{sec}$, IPSS mild or no symptoms, QOL improvement by $>-2$ grades and IVPP absent or grade $\mathrm{I}$.

\section{RESULTS}

1. The Incidence of Intravesical prostatic protrusion presented to our department in those who presented with Lower urinary tract symptoms due to benign prostatic hyperplasia is around 1: 5. (100:516).

2. Mean Age - 64 (100 men).

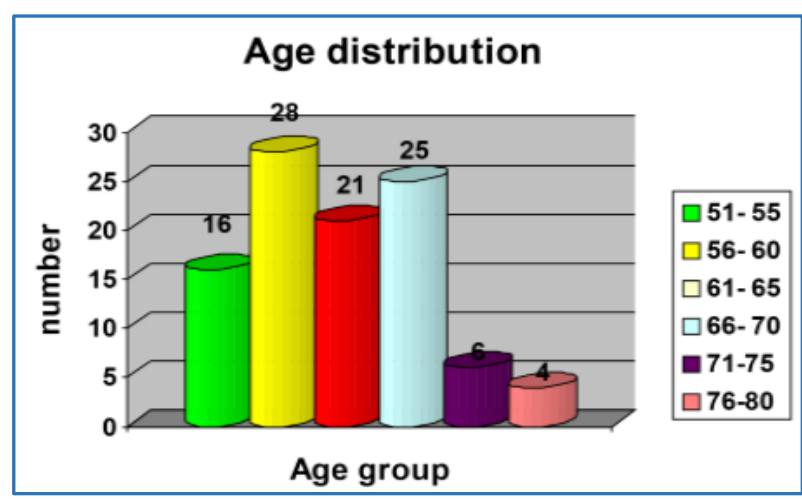

3. Total symptoms distribution

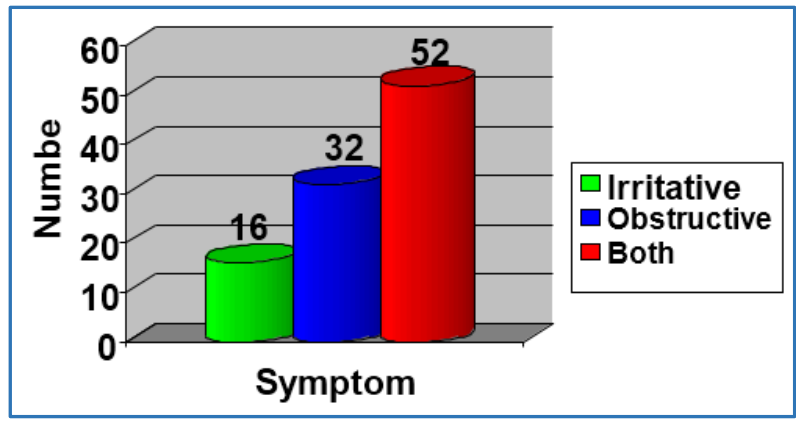


4. Intravesical prostatic protrusion Grade.

\begin{tabular}{|c|c|}
\hline Grade & Number \\
\hline I & 28 \\
\hline II & 43 \\
\hline III & 29 \\
\hline
\end{tabular}

5. IVPP and Correlation (all 100 patients).9,10

6. AUR and IVPP: 34 patients had acute urinary retention with Intravesical prostatic protrusion due to Benign prostatic hypertrophy (34\%); $85 \%$ of AUR pts. had IVPP $>5 \mathrm{~mm}$.

7. Cystoscopy.

\begin{tabular}{|c|c|}
\hline Lobes Enlarged & \% \\
\hline Lateral lobes & 12 \\
\hline Median lobe & 31 \\
\hline Both & 57 \\
\hline
\end{tabular}

8. Around 61 out of 100 underwent urodynamic evaluation (61\%).

9. Analysis of parameters.

10. Treatment.

\begin{tabular}{|c|c|c|}
\hline IVPP & Medical Rx & TURP \\
\hline Grade I & 12 & 16 \\
\hline Grade II & 11 & 32 \\
\hline Grade III & 5 & 24 \\
\hline
\end{tabular}

11. Comparing the medical and surgical treatment (12 cases of grade I put under medical Rx compared with 12 cases of grade I underwent TURP. Similarly, 11 cases of grade II and 5 cases of grade III are compared).

\section{Grade I}

\begin{tabular}{|c|c|c|}
\hline Parameters & Medical Rx & TURP \\
\hline IPSS Mean decrease by & 2 & 5 \\
\hline PF Mean increase & 1 & 3 \\
\hline IVPP Mean decrease & 0 & -- \\
\hline QOL Mean decrease & 0 & 0 \\
\hline Coefficient & -0.121 & 0.351 \\
\hline P value & $<0.231$ & $<0.034$ \\
\hline
\end{tabular}

\section{Grade II}

\begin{tabular}{|c|c|c|}
\hline Parameters & Medical Rx & TURP \\
\hline IPSS Mean decrease by & 2 & 7 \\
\hline PF Mean increase & 1 & 4 \\
\hline IVPP Mean decrease & 0 & -- \\
\hline QOL Mean decrease & 0 & 1 \\
\hline Coefficient & -0.211 & 0.381 \\
\hline P value & $<0.311$ & $<0.022$ \\
\hline
\end{tabular}

\section{Grade III}

\begin{tabular}{|c|c|c|}
\hline Parameters & Medical Rx & TURP \\
\hline IPSS Mean decrease by & 2 & 9 \\
\hline PF Mean increase & 1 & 5 \\
\hline IVPP Mean decrease & 0 & -- \\
\hline QOL Mean decrease & 0 & 1 \\
\hline Coefficient & 0.241 & 0.421 \\
\hline P value & $<0.351$ & $<0.014$ \\
\hline
\end{tabular}

12. AUR and IVPP.

\begin{tabular}{|c|c|c|c|}
\hline IVPP & AUR Pts. No. & Medical Rx & TURP \\
\hline Grade I & 5 & 2 & 3 \\
\hline Grade II & 11 & 1 & 10 \\
\hline Grade III & 18 & 1 & 17 \\
\hline
\end{tabular}

One pt. of Grade I IVPP voided well with medical treatment with follow-up period of 9 months. Other patients of AUR (of all grades IVPP) put under medical treatment failed trial voiding and later proceeded with TURP and succeeded trial voiding. All these patients had PFS and detrusor instability is ruled out in all cases.

\section{DISCUSSION}

The Incidence of Intravesical prostatic protrusion presented to our department in those who presented with Lower urinary tract symptoms due to benign prostatic hyperplasia is around 1:5 (100:516). The mean age is 64 (100 men). Majority of the patients are in the age group between 55 and 70 years; $52 \%$ of the patients presented with both obstructive and storage symptoms and the percentage of patients who presented only with obstructive symptoms is $32 \%$.

Majority of the patients are in Grade II IVPP (43\%), whereas the incidence of Grade I and Grade II patients is almost the same. All the Grades of IVPP are correlated with IPPS score, QOL index max, PVR and Prostatic volume and the significance is calculated using linear regression analysis and Pearson correlation coefficient. The increasing grades of IVPP are significantly directly correlated with IPSS score ( $\mathrm{r} 0.163, \mathrm{p}$ $<0.037$ ), Q-max (r $0.231 \mathrm{p}<0.027$ ) and Post-void residual ( $\mathrm{r}$ 0.331 $\mathrm{p}<0.031$ ). ${ }^{11}$ Among the three $\mathrm{Q}$-max is the best correlated one. The Prostatic volume $(\mathrm{p}<0.131)$ and QOL index $(p<0.053)$ do not correlate well with IVPP. 12

The percentage of the patients who presented with acute urinary retention is $34 \%$. Cystoscopy is done in all patients and majority of them had enlargement of both lateral and median lobes (57\%). Median lobe alone is presented as IVPP in $31 \%$ and lateral lobe alone is in $12 \%$. Majority of the grade II and III IVPP in TAUS showed trilobar prostatic enlargement in cystoscopy. 13

Urodynamics are done according to the "good urodynamic practices" recommended by the International Continence Society. Bladder Outlet Obstruction Index (BOOI), defined as the Detrusor's pressure at the maximum urinary flow (Pdet_Q-max) minus two times the maximum flow (Q-max): BOOI=pdet_qmax-2 x Qmax. Values below 20 were considered non-obstructed, between 20 and 40 inconclusive and higher than 40 , obstructed. But we have classified the values into two in this study whether obstructed or not obstructed, i.e. above 40 is obstructed and below 40 is not obstructed. Pressure flow study is done only in 61 patients (out of 100), as the Urodynamic machine (Delphi's TM Laborie) is available only from mid-2013 in our department; $71 \%$ of grade I, $78 \%$ of grade II and $88 \%$ of grade III IVPP had significant obstruction (BOOI $>40$ ) demonstrated with pressure flow study $(\mathrm{p}<0.011) .14$ The equivocal criteria in $\mathrm{PF}$ study (BOOI 20-40) is not considered in this study and BOOI $>40$ are considered as obstructed, whereas BOOI $<40$ are considered as non-obstructed. IPPS score (with the criteria $>21$ as significant), Q-max (with the criteria $>10 \mathrm{~mL} / \mathrm{sec}$ as significant) and PVR (with the criteria $>50 \mathrm{~mL}$ as significant) 
are significantly and directly correlated with the obstruction as demonstrated by the Pressure flow study. ${ }^{15}(\mathrm{P}<0.041$, $\mathrm{p}<0.031$ and $\mathrm{p}<0.041$ respectively), whereas the Prostatic volume and QOL index comparatively have lesser significance in assessing the obstruction. ${ }^{16,17}(\mathrm{P}<0.142$ and $\mathrm{p}<0.167$ respectively). Those patients presented with AUR after the period of mid-2013, made their catheter removed and proceeded with pressure flow study. With the urodynamic catheter in situ, they proceeded with voiding study and they voided minimally even though they did not void in the previously conducted Uroflowmetry machine.

Based on their significant criteria for obstruction as mentioned above and $2 * 2$ table mode statistical analysis, Qmax, PVR and IPSS have good positive predictive values (PPV 72,68 and 58 respectively) for assessing the severity of obstruction as confirmed by Pressure flow study.7,8 Among the three Q-max alone have a good negative predictive value (NPV 69), whereas others have similar NPV (around 44). IVPP along with Q-max and PVR is correlated for obstruction as demonstrated by PFS and analysed statistically using computer software packages SPSS (Statistical Package for Social Sciences) and Positive predictive value calculator 9.0. IVPP has a very good positive and negative predictive values $(78,73)$ and also a good accuracy rate $(78)$ in comparison with all other parameters in predicting the BO0.14 This is especially very significant in increasing grades of protrusion. In conclusion from this prospective analysis the IVPP correlates significantly with BOO; it should be used as one of the variables initially assessed in men with LUTS, being a less invasive (than pressure-flow studies in every patient) and a more costeffective way to stratify patients with LUTS for further management.

On the treatment aspect, those patients who are fit and willing for surgery and those patients indicated for surgery are proceeded with conventional TURP. Others are put under medical treatment. This comparison comes under Grade B recommendation of evidence, as this is level II evidence. Statistical and graphical analysis was performed using computer software packages SPSS (Statistical Package for Social Sciences) 12.0 for Windows and Microsoft Excel. No patient underwent open prostatectomy, as no one had prostatic volume greater than $75 \mathrm{cc}$.

Comparing the medical and surgical treatment $(12$ cases of grade I put under medical Rx compared with 12 cases of grade I underwent TURP. Similarly, 11 cases of grade II and 5 cases of grade III are compared). TURP showed a very good and significant mean decrease of IPSS and Q- $\max (\mathrm{p}<0.014$ to $0.034 ; \mathrm{r} 0.351$ to 0.421 ), whereas those who had medical treatment showed only a mean decrease of 2 in IPSS score and 1 in Q-max ( $\mathrm{p}<0.231$ to $0.351 ; r_{-} \_.241$ to _0.121) in all grades irrespectively. The patients who showed improvement in IPSS score with medical treatment had storage symptoms, especially before the treatment. There is no decrease in the measurement of IVPP in those patients with medical treatment, whereas TURP patients showed no demonstrable protrusion. The QOL index decreased by mean 1 in those underwent TURP, whereas the mean decrease is zero in those who had medical treatment. One pt. of Grade I IVPP voided well with medical treatment with follow-up period of 9 months. Other patients of AUR (of all grades IVPP) put under medical treatment failed trial voiding and later proceeded with TURP and succeeded trial voiding. All these patients had
PFS and detrusor instability is ruled out in all cases. So surgical intervention statistically proved to be superior to medical treatment in IVPP patients, especially most significant in Grade II and III IVPP patients.

\section{CONCLUSION}

1. The increasing grades of IVPP are significantly directly correlated with IPSS score max and post void residual. Among the three, Q-max is the best correlated one. The Prostatic volume and QOL index do not correlate well with IVPP. 18

2. IVPP has a very good positive and negative predictive value and also a good accuracy rate in comparison with all other parameters in predicting the B00. This is especially very significant in increasing grades of protrusion. Thus, the IVPP correlates significantly with BO0.19; it should be used as one of the variables initially assessed in men with LUTS being less invasive (than pressure-flow studies in every patient. ${ }^{20}$ ) and a more cost-effective way to stratify patients with LUTS for further management.

3. The surgical intervention statistically proved to be superior to medical treatment in IVPP patients, especially most significant in Grade II and III IVPP patients.

4. In patients presented with AUR along with IVPP surgery proved the efficacy for successful trial voiding. The patients failed medical treatment, voided successfully after TURP.

\section{REFERENCES}

1. Chia SJ, Heng CT, Chan SP, et al. Correlation of intravesical prostatic protrusion with bladder outlet obstruction. BJU Int 2003;91(4):371-4.

2. Schäfer W, Abrams P, Liao L, et al. Good urodynamic practices: uroflowmetry, filling cystometry, and pressureflow studies. Neurourol Urodyn 2002;21(3):261-74.

3. Franco G, Denunzio C, Leonardo C, et al. Ultrasound assessment of intravesical prostatic protrusion and detrusor wall thickness-new standards for non-invasive bladder outlet obstruction diagnosis? J Urol 2010;183(6):2270-4.

4. Kang M, Kim M, Choo MS, et al. Urodynamic features and significant predictors of bladder outlet obstruction in patients with lower urinary tract symptoms/benign prostatic hyperplasia and small prostate volume. Urology 2016;89:96-102.

5. Dicuio M, Pomara G, Vesely S, et al. The use of prostatic intravesical protrusion correlated with uroflowmetry: a new method to measure obstruction in patients with LUTS due to BOO without using P/F studies. Arch ital urol andrl 2005;77(1):50-3.

6. Yuen JS, Ngiap JT, Cheng CW, et al. Effects of bladder volume on transabdominal ultrasound measurements of Intravesical prostatic protrusion and volume. Int J Urol 2002;9(4):225-9.

7. Netto NR, D'Ancona CA, DeLima ML. Correlation between the international prostatic symptom score and a pressureflow study in the evaluation of symptomatic benign prostatic hyperplasia. J Urol 1996;155(1):200-2.

8. ElDin KE, Kiemeney LA, De Wildt MJ, et al. The correlation between bladder outlet obstruction and lower urinary tract symptoms as measured by the international prostate symptom score. J Urol 1996;156(3):1020-5. 
9. Din EK, Kiemeney LA, DeWildt MJ, et al. Correlation between uroflowmetry, prostate volume, post void residue, and lower urinary tract symptoms as measured by the international prostate symptom score. Urology 1996;48(3):393-7.

10. Tan YH, Foo KT. Intravesical prostatic protrusion predicts the outcome of a trial without catheter following acute urine retention. J Urol 2003;170(6 Pt 1):2339-41.

11. Keqin Z, Zhishun X, Jing Z, et al. Clinical significance of intravesical prostatic protrusion in patients with benign prostatic enlargement. Urology 2007;70(6):1096-9.

12. Lim KB, Ho H, Foo KT, et al. Comparison of intravesical prostatic protrusion, prostate volume and serum prostatic-specific antigen in the evaluation of bladder outlet obstruction. Int J Urol 2006;13(12):1509-13.

13. Bantis A, Zissimopoulos A, Kalaytzis C, et al. Correlation of serum prostate specific antigen, the volume and the intravesical prostatic protrusion for diagnosing bladder outlet obstruction in patients with benign prostate hyperplasia. Hell J Nucl Med 2007;10(2):138-43.

14. Nose H, Foo KT, Lim KB, et al. Accuracy of two non-invasive methods of diagnosing bladder outlet obstruction using ultrasonography: intravesical prostatic protrusion and velocity-flow video urodynamics. Urology 2005;65 (3): 493-7.
15. Jepsen JV, Bruskewitz RC. Comprehensive patient evaluation for benign prostatic hyperplasia. Urology 1998;51(4A Suppl):13-8.

16. Steele GS, Sullivan MP, Sleep DJ, et al. Combination of symptom score, flow rate and prostate volume for predicting bladder outflow obstruction in men with lower urinary tract symptoms. J Urol 2000;164(2):344-8.

17. Grossfeld GD, Coakley FV. Benign prostatic hyperplasia: clinical overview and value of diagnostic imaging. Radiol Clin North Am 2000;38(1):31-47.

18. Mastrigt RV, Pel JJM. Towards a non-invasive urodynamic diagnosis of intravesical obstruction. BJU Int 1999;84:195203.

19. Kuo HC. Clinical prostate score for diagnosis of bladder outlet obstruction by prostate measurements and uroflowmetry. Urology 1999;54(1):90-6.

20. Abrams PH, Griffiths DJ. The assessment of prostatic obstruction from urodynamic measurement and from residual urine. Br J Urol 1979;51(2):129-34. 